\title{
Design and Baseline Data from the Vanguard of the Comparison of Depression Interventions after Acute Coronary Syndrome (CODIACS) Randomized Controlled Trial
} \author{
$\mathbf{P h D}^{\mathrm{h}}$, and Karina W. Davidson, $\mathbf{P h D}^{\mathrm{a}}$ \\ ${ }^{a}$ Columbia University Medical Center, New York, NY \\ bWashington University School of Medicine, St. Louis, MO \\ cUniversity of North Carolina, Chapel Hill \\ dNational Heart Lung and Blood Institute, Washington, DC \\ eMcGill University, Montreal, Quebec, Canada \\ fUniversity of Pennsylvania, Philadelphia \\ 9Mayo Clinic, Rochester, MN \\ hUniversity of Montreal, Montreal, Quebec, Canada \\ 'Emory University School of Medicine, Atlanta, GA
}

William Whang, MD, MS ${ }^{\mathrm{a}}$, Matthew M. Burg, PhD ${ }^{\mathrm{a}}$, Robert M. Carney, PhD ${ }^{\mathrm{a}}$, Kenneth E. Freedland, $\mathrm{PhD}^{\mathrm{b}}$, J. Thomas Bigger, $\mathbf{M D}^{\mathrm{a}}$, Diane Catellier, $\mathrm{DrPH}^{\mathrm{c}}$, Susan Czajkowski, $\mathbf{P h D}^{\mathrm{d}}$, Nancy Frasure-Smith, PhDe,h, Donald C. Haas, MD, MPH ${ }^{f}$, Allan S. Jaffe, MDg, Francois Lespérance, MD $^{\mathrm{h}}$, Vivian Medina, MSWa ${ }^{\mathrm{a}}$, Joan Duer-Hefele, RN ${ }^{\mathrm{a}}$, Gabrielle A. Osorio, BS ${ }^{\mathrm{a}}$, Faith Parsons, BS ${ }^{a}$, Peter A. Shapiro, MD ${ }^{a}$, David S. Sheps, MD', Viola Vaccarino, MD,

\section{Abstract}

This paper describes the rationale and design of the vanguard for the Comparison of Depression Interventions after Acute Coronary Syndrome (CODIACS), a multicenter, randomized, controlled trial of a patient preference-based, stepped care protocol for persistent depressive symptoms after acute coronary syndrome (ACS). The overall aim of the vanguard phase was to determine whether the patient-preference, stepped care protocol, which is based on the intervention used in the recent Coronary Psychosocial Evaluation Studies (COPES) trial, was feasible in patients with recent ACS who were recruited from 5 geographically diverse sites. Innovative design features of this trial include randomization to either initial patient-preference of treatment or to a referred care arm in which the primary care provider decided upon care. Additionally, delivery of psychotherapy was accomplished by telephone, or webcam, depending upon patient preference. The vanguard phase provides estimates of eligibility and screening/enrollment ratios, patient acceptance of screening, and retention. In this report, we describe the innovative features and the baseline results of the vanguard phase of CODIACS. The data from this vanguard study will be used to finalize

\footnotetext{
(C) 2012 Elsevier Inc. All rights reserved.

Corresponding author: Karina Davidson, PhD, PH9-314, 622 West $168^{\text {th }}$ St, New York, NY 10032. Telephone: 212-342-4486. Fax 212-305-3431. kd2124@mail.cumc.columbia.edu.

Publisher's Disclaimer: This is a PDF file of an unedited manuscript that has been accepted for publication. As a service to our customers we are providing this early version of the manuscript. The manuscript will undergo copyediting, typesetting, and review of the resulting proof before it is published in its final citable form. Please note that during the production process errors may be discovered which could affect the content, and all legal disclaimers that apply to the journal pertain.
} 
planning for a large, phase III clinical trial designed to evaluate the effect of treatment on depressive symptoms, coronary events, and death.

\section{Keywords}

clinical trial; depression; acute coronary syndrome

\section{INTRODUCTION}

Despite the well-documented risk of recurrent cardiac events in acute coronary syndrome (ACS) patients with depressive symptoms [1,2], there is little evidence that treatment of depressive symptoms improves cardiac outcomes. The largest randomized trial thus far, the Enhancing Recovery in Coronary Heart Disease Patients (ENRICHD) trial, enrolled 2481 post-myocardial infarction (MI) patients with depression or low social support and found that cognitive behavior therapy, supplemented as needed with treatment with a selective serotonin reuptake inhibitor, had no effect on the combined end point of recurrent MI or death [3]. This first trial offered many lessons and helpful directions for new trials in this research area.

Recently, the Coronary Psychosocial Evaluation Studies (COPES) randomized trial incorporated several strategies suggested by ENRICHD, and that were designed to increase the probability of demonstrating benefit from an intervention to reduce depressive symptoms in post-ACS patients [4]. Instead of relying on a single treatment, COPES used a stepped care approach that provided brief, problem-focused psychotherapy and/or pharmacotherapy to reduce depressive symptoms. Also, a model based on patient preference was implemented to encourage patient acceptance of and participation in the treatment. Finally, because of the spontaneous improvement of symptoms often seen in the first few weeks after ACS, COPES recruited patients with depressive symptoms that persisted for at least 3 months after the onset of ACS.

In COPES, 157 post-ACS patients with elevated depressive symptoms were assigned to stepped care or usual care. At 9 months, 54\% of 80 patients in the stepped care group reported that their mental health care was excellent or very good, compared with $19 \%$ of 77 patients in the usual care group $(P<.001)$ [4]. In addition, depressive symptoms as measured by the Beck Depression Inventory (BDI)[5] total score improved 3.8 more points in the stepped care group than in the usual care group, representing a standardized effect size (Cohen $d$ ) of 0.59. Although the sample size was limited in COPES, there were fewer major adverse cardiac events, i.e., nonfatal MI or hospitalization for unstable angina in the stepped care group than in the usual care group (4\% versus 13\%, $P=.047$ )[4]. The improvement in cardiac outcomes associated with depressive symptoms treatment raised the possibility that the COPES intervention strategy could offer a promising model for a larger, adequately powered trial.

The experience of the COPES trial, which recruited patients from hospitals in New York and Connecticut, led us to undertake a multicenter, single-blind, parallel-group, feasibility/ vanguard study of the depressive symptom intervention tested in COPES. The vanguard phase of the Comparison of Depression Interventions After Acute Coronary Syndrome (CODIACS) was designed to determine the feasibility and effectiveness of a national, 5-site, randomized trial comparing the COPES stepped care protocol, modified to facilitate dissemination and implementation across geographically diverse centers, with referred care for post-ACS patients. Patients with an ACS event and comorbid depressive symptoms were recruited from a network of hospitals connected with 5 field centers (Columbia University, 
New York, NY; Washington University, St Louis, MO; University of Pennsylvania, Philadelphia; Emory University, Atlanta, GA; and Yale University, New Haven, CT). In the study, 150 participants were randomly assigned to receive either stepped care or referred care between 2-6 months after the ACS event, and the care was delivered over 6 months. This study aimed to address whether the COPES intervention can be delivered feasibly and effectively in multiple clinical centers with different practice models. Estimates of screening/recruitment ratio, patient acceptance of screening, retention, and improvement in depression symptoms will be used to finalize the estimates and planning for the large phase III CODIACS clinical trial.

\section{TREATMENT RATIONALE}

Designing a clinical trial that tests an intervention to reduce depressive symptoms after ACS poses several challenges. For instance, depressive symptoms after ACS are associated with reduced adherence to therapy [6], which might result in lower rates of treatment completion. In the Canadian Cardiac Randomized Evaluation of Antidepressant and Psychotherapy Efficacy (CREATE) study of interpersonal psychotherapy and citalopram, 19\% of randomized patients did not complete their allocated treatment [7], and in ENRICHD, 23\% of patients randomized to intervention did not complete at least 6 sessions of cognitive behavioral therapy [3]. In the Sertraline Antidepressant Heart Attack Randomized Trial (SADHART), $28.5 \%$ of patients in the sertraline group and $25.1 \%$ of patients in the placebo group discontinued the trial; importantly, the difference between the 2 arms was not statistically significant [8]. In COPES, $16 \%$ of patients randomized to intervention did not receive any study treatment, and a further $9 \%$ terminated treatment before their study provider advised it [4]. While these completion rates may be similar in magnitude to those in depression trials with noncardiac patients, they may not be sufficient to test if a reduction in depression symptoms improves medical prognosis. Another challenge is that, among individuals whose depression or depressive symptoms are identified during or immediately after an ACS, spontaneous improvement occurs in as many as 50\% of cases, depending on the cohort [9]. This would be expected to reduce the ability to detect a difference in depression or depressive symptom outcome between the intervention and control groups.

Several innovative features were tested in this vanguard to determine whether they should be incorporated into the larger, phase III CODIACS trial: (1) administration of an intervention (psychotherapy or medication) based on patient preference; (2) delivery of psychotherapy by telephone or webcam, again, based on patient preference; (3) delay of initial depressive symptom assessment for several months after hospitalization for an ACS, to exclude patients whose depressive symptoms spontaneously remit after ACS; and (4) use of the Beck Depression Inventory (BDI) [5] for depression screening, rather than a full psychiatric diagnostic interview or the Patient Health Questionnaire (PHQ-9)[10]. These design features are described below, and their usefulness is evaluated in the Discussion.

The patient-preference, stepped care algorithm could be tested against many different depression algorithms or control groups, with different implications for the conduct of the trial [11]. A recent advisory from the American Heart Association that was also endorsed by the American Psychiatric Association recommended screening, referring, and treating depression when detected in patients with coronary disease [12]. It specifically recommended administering a self-report depressive symptom screening questionnaire to patients with coronary heart disease (CHD) and referring those whose screening results are positive to a professional qualified to diagnose and manage their depressive symptoms. Even recently, depression or elevated depressive symptoms have been noted to be underrecognized in ACS patients [13]. The advisory notes that cardiologists should be aware that depressed patients are at increased risk of cardiac morbidity and mortality and that they 
should aggressively manage depressed patients' treatment compliance, engagement in cardiac rehabilitation, and lifestyle modification plans. As this standard of depression care is recommended for all CHD patients, we employed it as our active comparator in the referred care arm.

\section{METHODS}

We obtained approval to conduct the study from the institutional review board at each participating institution. To identify participants for CODIACS, we reviewed clinic charts, inpatient admission logs, and cardiac catheterization laboratory records for ACS. Consent for participation in the trial was obtained from potential participants in a 2 stage process, once prior to a screening interview and again prior to randomization. Initial screening for depressive symptoms was performed with the BDI, a well-validated self-report questionnaire. For those who qualified for the study based on their BDI score, we performed additional screening to determine medical eligibility. Eligible patients who agreed to participate were randomized to receive either referred care or stepped care. Initial treatment in the stepped care arm was according to patient preference, and reviews were performed approximately 6 and 12 weeks after randomization to assess progress and adjust or step up the treatment protocol. Repeated assessments of depressive symptoms by the BDI were performed in both the stepped care and referred care arms at 2, 4, and 6 months after randomization by assessors masked to participants' randomization.

\section{Recruitment and enrollment}

The eligibility criteria for CODIACS were: (1) age at randomization of at least 35 years1; (2) fluent in English or Spanish; (3) ACS, i.e.,unstable angina or MI within the past 2 to 6 months; and (4) depressive symptoms 2 to 6 months after ACS.

\section{ACS Eligibility Criteria}

Patients with acute ST-segment elevation MI, non-ST-segment elevation MI, or documented unstable angina were enrolled. Enrolling both patients with MI and unstable angina increased the generalizability and reach of this trial, as depressive symptoms are prevalent in both subgroups and pose similar prognostic risks. MI was defined by ischemic chest pain lasting 20 minutes or longer and acute rise in serum levels of cardiac biomarkers that met the criteria of the Task Force for the Universal Definition of Acute MI [14].

Unstable angina was defined by ischemic symptoms lasting 20 minutes or longer with recent onset or with an accelerating pattern, or episodes at rest, and at least one of the following: ischemic electrocardiographic abnormalities, an angiogram indicative of coronary artery disease during the current hospital admission, and/or a documented history of coronary artery disease. To ensure the comparability of criteria for the diagnosis of acute MI across sites, each site was asked to provide the name of its troponin assay and the cutoff value used for the diagnosis of MI.

\section{Depressive symptom criteria}

Depressive symptoms were assessed 2 to 6 months after the index ACS event. Patients were eligible for inclusion if they had a BDI score of $\geq 10$ twice at least 2 weeks apart, or a BDI score of $\geq 15$ once. The criterion of 2 BDI scores $\geq 10$ was used to ensure that depressive symptoms persisted. Previous data suggests that patients with a BDI score $\geq 15$ obtained 2 to

\footnotetext{
${ }^{1}$ The limit of 35 years of age was set by one of the site IRBs. This committee required that either multiple urine samples be obtained from all female study candidates (regardless of age) and tested for pregnancy status, or that we increase the minimum age of study eligibility for female participants. We chose to raise the inclusionary age to 35 years rather than deal with the complexities of transporting and testing urine specimens and reporting results back to sites. This was not considered to be feasible for the larger trial.
} 
6 months after ACS are very unlikely to remit spontaneously, so only 1 score above this level was required for eligibility. The BDI was chosen because it is the measure with the most extensive evidence of predictive validity (that is, for predicting MI recurrence and death) compared to all other self-report depression measures. It was used in 13 of the 22 studies reviewed in a large meta-analysis testing the prognostic association of depression or depressive symptoms with cardiovascular events and death [15]. Although we have recommended use of the PHQ-9 in future trials [16], we still find little evidence that the PHQ-9 is associated with adverse medical outcomes, despite the use of this measure in newer cohort studies. Also, the PHQ-9 was used by the staff to step depressive symptom care. If the PHQ-9 had also been used as a screening and outcome measure, this would have produced differential assessment between trial arms (i.e., the stepped care group would have had more administrations of the PHQ-9 than the referred care group).

Finally, we chose not to use a standardized psychiatric interview to diagnose clinical depressive episodes, for a number of reasons. First, even subsyndromal levels of depressive symptoms place post-ACS patients at increased risk for cardiac event recurrence or mortality $[2,15]$. Second, there are pragmatic barriers to administering a psychiatric interview over the telephone that do not exist when requesting a self-report questionnaire in person or over the web, e.g., scheduling/time-zone conflict, need for privacy for potential participant, need for a telephone. Finally, some local IRBs have recently found that clinically-diagnosed depression, once identified, must be treated, and regard randomization to any type of control condition as unethical.

\section{Exclusion criteria}

The exclusion criteria for CODIACS were: (1) presence of a noncardiac condition likely to cause death within 1 year; (2) lack of availability for intervention or follow-up (e.g., plans to move); (3) cognitive impairment; (4) need for immediate psychiatric intervention (i.e., requirement for hospitalization or psychiatric intervention within 72 hours); (5) suicidal ideation; (6) major psychiatric comorbidity (current or past), including active psychosis, bipolar disorder, or severe personality disorder; (7) active substance abuse or dependency; (8) impaired renal function (serum creatinine level $\geq 2.0 \mathrm{mg} / \mathrm{dL}$ ) or moderate or severe liver disease (e.g., esophageal varices, portal hypertension, encephalopathy, gastrointestinal bleeding); or (9) current participation in another clinical trial.

\section{Treatment team}

Stepped care was delivered by a team of professionals including a centralized Problem Solving Therapy (PST) specialist, a local site case coordinator, a local team physician or advanced practice registered nurse responsible for prescribing and managing antidepressant medications, and other local support personnel as needed. These staff members met on a weekly basis with a central study psychiatrist and clinical psychologist to discuss participant issues, and to jointly determine stepped care decisions within the patient-chosen therapy; this was called our clinical review team. The participant's treating cardiologist or other primary physician was informed by letter about the patient's participation in the trial and about his or her depression treatment, both at the beginning and end of treatment.

\section{Stepped care group}

Those assigned to the stepped care group had a choice of PST, pharmacotherapy, or a combination of the two (Figure 1). Participants who were randomized to the stepped care group had a 15-minute information session on the benefits and drawbacks of each type of therapy and medication, after which the preferred treatment was chosen. If patients chose to do so, they were permitted to indicate their preference at a later session after they had spoken to family members or reflected on the new information. The treatment incorporated 
essential components from the Improving Mood-Promoting Access to Collaborative Treatment (IMPACT) trial [9].

\section{Problem solving therapy}

PST, a protocol-driven and problem-focused form of cognitive-behavioral therapy, was an option for participants in the stepped care intervention arm. PST teaches patients how to systematically solve psychosocial problems that can perpetuate depression $[9,10]$ and is tailored to the specific problems faced by individual participants. We selected this type of therapy because it is easily standardized, is time limited, is readily accepted by most patients, requires little psychological insight on the part of the patient, and can be administered by trained social workers, nurses, psychologists, and other allied health care professionals.

In the vanguard phase of CODIACS, the PST intervention was delivered centrally by treatment core staff at the coordinating center at Columbia University. For participants who were randomized to receive stepped care and who chose PST, the initial assessments were performed via webcam between the recruiting site and the coordinating center, and subsequent sessions occurred either by webcam or by telephone, depending on patient preference [17]. PST sessions initially occurred weekly, with each session lasting approximately 30 to 45 minutes. As depressive symptoms were reduced, session frequency was decreased to every 2 weeks, and if progress continued, the frequency was further reduced, following previously tested guidelines [18]. If depressive symptoms were not reduced, therapy sessions were given more frequently.

\section{Pharmacotherapy}

Options within stepped care included sertraline, citalopram, or bupropion as first-line treatments. Participants who chose pharmacotherapy were interviewed by the site physician or nurse practitioner to establish the appropriate medication and dosage based on the participants's prior medication history and current symptoms. During the trial medications were initiated by study clinicians. The study did not provide any prescription medications for participants in either treatment arm; instead, they were asked to obtain the medications from a pharmacy. Dosing followed standard clinical practice, with participants prescribed what was identified as the minimally effective dose when treatment was initiated (e.g., for sertraline, $50 \mathrm{mg}$ ), although some patients were prescribed lower doses if medically indicated (e.g., frailty) or to limit adverse effects. Participants were seen in person by a local study physician or nurse at 1- to 2-week intervals for dose titration, and thereafter every 3 to 5 weeks as needed. The focus of these treatment contacts was to evaluate depressive symptoms and to detect adverse effects of medication. Participants who chose pharmacotherapy but who had already been prescribed a nonstudy antidepressant had their treatment coordinated with the prescribing physician or practitioner.

\section{Depressive symptom monitoring and stepped care decisions during intervention}

The PHQ-9 was used to monitor depressive symptoms, to determine whether the participant's symptoms had improved relative to baseline in the stepped care intervention (Figure 1). A centralized treatment committee consisting of a clinical psychiatrist, a clinical psychologist, a social worker, and the study treatment providers discussed individual cases at 6 to 8 weeks post-randomization. The minimum improvement criterion for continuing a particular treatment was based on the participant's initial PHQ-9 score; the larger the initial score, the larger the improvement in PHQ-9 that was required to remain at a particular step. For instance, participants with an initial PHQ-9 score of 5 to 10 required a $30 \%$

improvement in their score to remain at their current step, and those with an initial score of 20 or greater required a $60 \%$ improvement. If the minimum improvement criterion had not 
been achieved, the review committee offered suggestions for subsequent treatment, and additional or alternative treatments were subsequently initiated with patient agreement. Additional treatment modifications were made 6 to 8 weeks later, with the objective of treating the depression to remission by the end of the intervention. Relapse monitoring and maintenance therapy continued for the 6-month duration of the trial.

Patients could be referred to an outside mental health specialist if they were not responding to the study intervention or needed specialized care (e.g., addiction, severe trauma). Two participants in CODIACS were referred for such care during the study. In addition, 1 participant was referred for neuropsychological assessment, and 1 was referred to a memory disorders clinic.

\section{Referred care group}

In the referred care arm, the participant's primary care provider was notified in writing of the finding of elevated depressive symptoms and encouraged to implement a providerpreferred depression treatment [12]. Depending on the provider's evaluation of the participant, he or she elected to defer depression treatment, to initiate it, or to refer the participant to a mental health specialist.

\section{Randomization and masking}

The randomization sequence was created by computer and was stratified by site and by previous antidepressant use, chosen due to the improved depression response that was observed in SADHART in patients with prior major depressive disorder [19]. This was a single-blind study, as follow-up staff, but not participants, were masked to the group assignment. A separate staff member who was not involved in follow-up called into the automated telephone system to receive the randomization assignment and then informed the participant of the treatment arm.

\section{End point adjudication}

The primary outcomes for CODIACS were yield and trial completion rates, and the secondary end point was change in BDI score at 6 months. For the future phase III trial, the primary end point will be nonfatal MI and/or all-cause mortality. The current study is a vanguard for the larger trial and did not have adequate power to test the effect of the intervention on this end point.

Participants were assessed via telephone at 2 and 4 months after randomization, and a final visit occurred 6 months after randomization. Contact information for family and/or friends was collected in case a participant moved during the 6-month trial. At each follow-up session, participants were assessed for depressive symptoms using the BDI and were asked if they had been hospitalized since the previous visit. Hospital systems were actively surveyed for events, and for each hospitalization, hospital records were requested. An adjudication committee, blind to treatment status, classified and adjudicated the primary reason for each hospitalization. For participants who could not be contacted or who were reported by a relative to be deceased, we searched the Social Security Index and the National Death Index to verify vital status, and we obtained death certificates. Participants in each arm received the same number and duration of assessment contacts.

The field center team ensured that unexpected adverse events were managed appropriately and reported them to the local institutional review board. The Data Safety and Monitoring Board reviewed unexpected adverse events annually. 


\section{Safety protocol}

During screening, we excluded patients found to be actively suicidal, and we immediately referred these patients for further clinical assessment and emergency treatment if appropriate. Patients who were ineligible were reported to their health care providers so that appropriate treatment could be offered. A score of $\geq 2$ on the BDI item 9 (suicidality) initiated the same safety protocol as for patients enrolled in the trial. When initiated, this protocol required notification of a local site study psychologist or psychiatrist, and based on the patient's presentation and history, determination as to whether it was safe for the patient to participate in the study and whether the patient's physician should be notified. These protocols were followed for both in-person and telephone contacts.

\section{Sample size, power calculations, and data analysis}

The size of the vanguard phase of CODIACS was similar to that of COPES and was feasible to accrue within the logistical constraints of a 2-year funding period with recruitment conducted at 5 sites. This feasibility study was not powered to detect differences in depression, or in MI and/or mortality rate. Detecting differences in hard medical end points and clinically meaningful depressive symptom reduction will be the aim of the larger trial. The proposed sample size of 150 patients was set to allow an $80 \%$ chance of detecting a difference in depression scores between groups of 0.46 SD-units (e.g., approximately 3.8point difference in BDI) assuming 5\% loss to follow-up. Group differences in depression outcomes will be evaluated using multilevel repeated-measures modeling procedures to generate full-information maximum likelihood estimates and to compare the estimated effect size of a multicenter implementation of the stepped care intervention with that reported by the regional COPES trial. By including all participants and all available data, this approach yields intent-to-treat estimates that are valid under the assumption that the missing data are missing at random, conditional on the observed data [20,21]. Clinical endpoint data, including number of recurrent MI, number or urgent revascularizations, number of deaths, and number of hospitalization days, will be used to help project the length of follow-up and number of participants and associated events that will be needed to have power (> 80\%) to detect a $20 \%$ difference between groups in the phase III study.

\section{BASELINE DEMOGRAPHICS}

Figure 2 presents the CONSORT diagram for CODIACS. Recruitment took place from March 2010 through June 2011. The last follow-up visit was January 2012. Among 724 medically eligible patients, $327(45 \%)$ refused to be screened for the trial and 247 (34\%) were found not to be persistently depressed or to be ineligible based on other criteria stated above. Among the 150 patients who were randomized to care, 73 (49\%) were assigned to stepped care and $77(51 \%)$ to referred care. Among the 73 participants in the stepped care arm, $14(19 \%)$ did not complete their full treatment. Six participants in each of the referred care and stepped care arms were lost to follow-up at 6 months, and so had missing depressive symptom and potential nonfatal event data. Vital status will be ascertained through a search of the National Death Index for all 150 participants.

Table 1 provides baseline demographic and clinical characteristics for the participants who were randomized to care. The average age of CODIACS participants was 60 years, and 63 (42\%) participants were women. More than $50 \%$ of the patients randomized to care in CODIACS qualified medically on the basis of MI, as opposed to $22 \%$ to $26 \%$ of the patients in COPES. This difference is likely due in part to the use of more sensitive troponin cutoff points for MI used in this vanguard of CODIACS.

Table 2 provides a description of the differing ways the sites identified and enrolled participants. As expected, screening efficiency, defined according to the number of 
participants enrolled as a percentage of individuals approached, ranged widely among the sites, from $11 \%$ to $80.0 \%$. The most efficient site also invested the most human labor in their recruitment strategy; rather than relying on automated, electronic searches, the site nurse reviewed all possible cardiac admissions, and then individually reviewed each chart before approaching the physician or participant. This strategy led to the most efficient yield, but time spent by the nurse was the most of any site. The second most efficient site ( $42 \%$ yield) waited for cardiac rehabilitation to be completed, and then examined the depression scores recorded in the chart. This led to a very reasonable yield rate. The private practice clinic did not yet have an electronic medical record, and used scheduled visits for the initial identification of participants; this was the most inefficient strategy.

\section{COMMENT}

Both COPES and the vanguard phase of CODIACS will guide design considerations for the future CODIACS phase III trial of treatment of depressive symptoms in cardiac patients. There are 3 major differences between the design of the vanguard phase of the CODIACS and COPES trials. First, assessment of depressive symptoms for inclusion of each potential participant in CODIACS was performed during a 2-week period 2 to 6 months after the hospitalization for the index ACS. In COPES, inclusion depended on elevated depressive symptoms from assessments at 2 different periods, within 1 week of ACS hospitalization and 3 months later [4]. One might speculate that some of the patients who qualified for CODIACS became depressed only in the months after their index ACS event and were not depressed at the time of their ACS. Therefore, they may not have met the same definition of persistent depression used in COPES. In CODIACS, delivery of PST in the depression intervention arm was centralized and performed via webcam or telephone, whereas in COPES the PST was delivered locally by the participating centers. Third, substantially more CODIACS participants had an MI as their qualifying ACS than did participants in COPES. We expect that the first 2 of these differences will make it easier for CODIACS to scale up to a larger trial, assuming that the beneficial effect of treatment on depression is replicated; the third distinction may make recruitment more difficult, or may be neutral with respect to recruiting efficiency.

\section{Usefulness of different recruitment strategies}

In examining the different strategies employed to recruit participants, we believe that future sites should have an electronic medical record, show capability to set up automatic alerts for troponins and ACS admissions and discharges, and have some way of asking participants if they are initially interested in being screened through mail, telephone, or emails. Sites that were required by their IRB to obtain written physician consent, and before in-person consent for screening, were the least efficient. Finally, as sites increasingly adopt the recent AHA advisory to screen for depression [12], searching for ACS patients with elevated depressive symptoms recorded in the electronic medical record will become an increasingly efficient strategy.

The completion of CODIACS is expected to help clarify other potential issues in designing a more definitive study. For instance, the 19\% dropout rate from complete treatment in the stepped care arm, in the context of similar attrition rates in other prior studies, raises the consideration that a phase III trial may benefit from use of either a 'wait and see' period for less depressed participants or a run-in phase to maximize the likelihood of trial completion. However, this would presumably reduce the generalizability of the results. Also, if the results of the vanguard of CODIACS demonstrate that depression treatment reduces cardiac events at a rate similar to that found in COPES, it may be possible to power a larger trial for a mortality end point, as the preponderance of prospective observational studies among ACS 
patients have documented a strong association between depression and all-cause mortality [1].

\section{CONCLUSION}

The vanguard phase of CODIACS provided data on the feasibility of expanding to multiple sites the depression intervention studied in COPES. Important information gained from the vanguard phase of CODIACS included: (1) the efficiency of enrollment of post-ACS patients with persistent depressive symptoms from various recruiting strategies; (2) the ability to deliver an intervention based on patient preference nationally; and (3) the use of PST as a nationwide treatment option. In addition, the delivery of PST by telephone or webcam from a centralized location broadens the numbers of recruiting sites that can participate and efficiently deliver PST.

\section{ABBREVIATIONS}

$\begin{array}{ll}\text { ACS } & \text { acute coronary syndrome } \\ \text { ENRICHD } & \text { Enhancing Recovery in Coronary Heart Disease Patients } \\ \text { MI } & \text { myocardial infarction } \\ \text { COPES } & \text { Coronary Psychosocial Evaluation Studies } \\ \text { BDI } & \text { Beck Depression Inventory } \\ \text { CHD } & \text { coronary heart disease } \\ \text { IMPACT } & \text { Improving Mood-Promoting Access to Collaborative Treatment } \\ \text { PST } & \text { problem solving therapy }\end{array}$

\section{References}

1. Nicholson A, Kuper H, Hemingway H. Depression as an aetiologic and prognostic factor in coronary heart disease: a meta-analysis of 6362 events among 146538 participants in 54 observational studies. Eur Heart J. 2006; 27:2763-2774. [PubMed: 17082208]

2. Barth J, Schumacher M, Herrmann-Lingen C. Depression as a risk factor for mortality in patients with coronary heart disease: a meta-analysis. Psychosom Med. 2004; 66:802-813. [PubMed: 15564343]

3. Berkman LF, Blumenthal J, Burg M, Carney RM, Catellier D, Cowan MJ, et al. Effects of treating depression and low perceived social support on clinical events after myocardial infarction: the Enhancing Recovery in Coronary Heart Disease Patients (ENRICHD) Randomized Trial. Jama. 2003; 289:3106-3116. [PubMed: 12813116]

4. Davidson KW, Rieckmann N, Clemow L, Schwartz JE, Shimbo D, Medina V, et al. Enhanced depression care for patients with acute coronary syndrome and persistent depressive symptoms: coronary psychosocial evaluation studies randomized controlled trial. Arch Intern Med. 2010; 170:600-608. [PubMed: 20386003]

5. Beck AT, Ward CH, Mendelson M, Mock J, Erbaugh J. An inventory for measuring depression. Arch Gen Psychiatry. 1961; 4:561-571. [PubMed: 13688369]

6. Rieckmann N, Kronish IM, Haas D, Gerin W, Chaplin WF, Burg MM, et al. Persistent depressive symptoms lower aspirin adherence after acute coronary syndromes. Am Heart J. 2006; 152:922927. [PubMed: 17070160]

7. Lesperance F, Frasure-Smith N, Koszycki D, Laliberte MA, van Zyl LT, Baker B, et al. Effects of citalopram and interpersonal psychotherapy on depression in patients with coronary artery disease: the Canadian Cardiac Randomized Evaluation of Antidepressant and Psychotherapy Efficacy (CREATE) trial. Jama. 2007; 297:367-379. [PubMed: 17244833] 
8. Lespérance F, Frasure-Smith N. Sertraline for Treatment of Depression in Acute Coronary Syndromes. JAMA: The Journal of the American Medical Association. 2002; 288:2403-2404.

9. Davidson KW, Rieckmann N, Lesperance F. Psychological theories of depression: potential application for the prevention of acute coronary syndrome recurrence. Psychosom Med. 2004; 66:165-173. [PubMed: 15039500]

10. Kroenke K, Spitzer RL, Williams JB. The PHQ-9: validity of a brief depression severity measure. J Gen Intern Med. 2001; 16:606-613. [PubMed: 11556941]

11. Freedland KE, Mohr DC, Davidson KW, Schwartz JE. Usual and unusual care: existing practice control groups in randomized controlled trials of behavioral interventions. Psychosom Med. 2011; 73:323-335. [PubMed: 21536837]

12. Lichtman JH, Bigger JT Jr, Blumenthal JA, Frasure-Smith N, Kaufmann PG, Lesperance F, et al. Depression and coronary heart disease: recommendations for screening, referral, and treatment: a science advisory from the American Heart Association Prevention Committee of the Council on Cardiovascular Nursing, Council on Clinical Cardiology, Council on Epidemiology and Prevention, and Interdisciplinary Council on Quality of Care and Outcomes Research: endorsed by the American Psychiatric Association. Circulation. 2008; 118:1768-1775. [PubMed: 18824640]

13. Smolderen KG, Spertus JA, Reid KJ, Buchanan DM, Krumholz HM, Denollet J, et al. The Association of Cognitive and Somatic Depressive Symptoms With Depression Recognition and Outcomes After Myocardial Infarction. Circulation: Cardiovascular Quality and Outcomes. 2009; 2:328-337. [PubMed: 20031858]

14. Thygesen K, Alpert JS, White HD, Jaffe AS, Apple FS, Galvani M, et al. Universal definition of myocardial infarction. Circulation. 2007; 116:2634-2653. [PubMed: 17951284]

15. van Melle JP, de Jonge P, Spijkerman TA, Tijssen JG, Ormel J, van Veldhuisen DJ, et al. Prognostic association of depression following myocardial infarction with mortality and cardiovascular events: a meta-analysis. Psychosom Med. 2004; 66:814-822. [PubMed: 15564344]

16. Davidson KW, Kupfer DJ, Bigger JT, Califf RM, Carney RM, Coyne JC, et al. Assessment and treatment of depression in patients with cardiovascular disease: National Heart, Lung, and Blood Institute Working Group Report. Psychosom Med. 2006; 68:645-650. [PubMed: 17012516]

17. Simon GE, Ludman EJ, Tutty S, Operskalski B, Von Korff M. Telephone psychotherapy and telephone care management for primary care patients starting antidepressant treatment: a randomized controlled trial. JAMA. 2004; 292:935-942. [PubMed: 15328325]

18. Unutzer J, Katon W, Callahan CM, Williams JW Jr, Hunkeler E, Harpole L, et al. Collaborative care management of late-life depression in the primary care setting: a randomized controlled trial. JAMA. 2002; 288:2836-2845. [PubMed: 12472325]

19. Glassman AH, Bigger JT, Gaffney M, Shapiro PA, Swenson JR. Onset of major depression associated with acute coronary syndromes: relationship of onset, major depressive disorder history, and episode severity to sertraline benefit. Arch Gen Psychiatry. 2006; 63:283-288. [PubMed: 16520433]

20. Wothke, W. Longitudinal and multigroup modeling with missing data. In: Little, T.; Schnabel, K.; Baumert, J., editors. Modeling Longitudinal and Multilevel Data: Practical Issues, Applied Approaches, and Specific Examples. Mahwah, NJ: Lawrence Erlbaum Associates Inc; 2000. p. 219-240.

21. Little, R.; Rubin, D. Statistical analysis with missing data. New York, NY: John Wiley \& Sons Inc; 1987. 


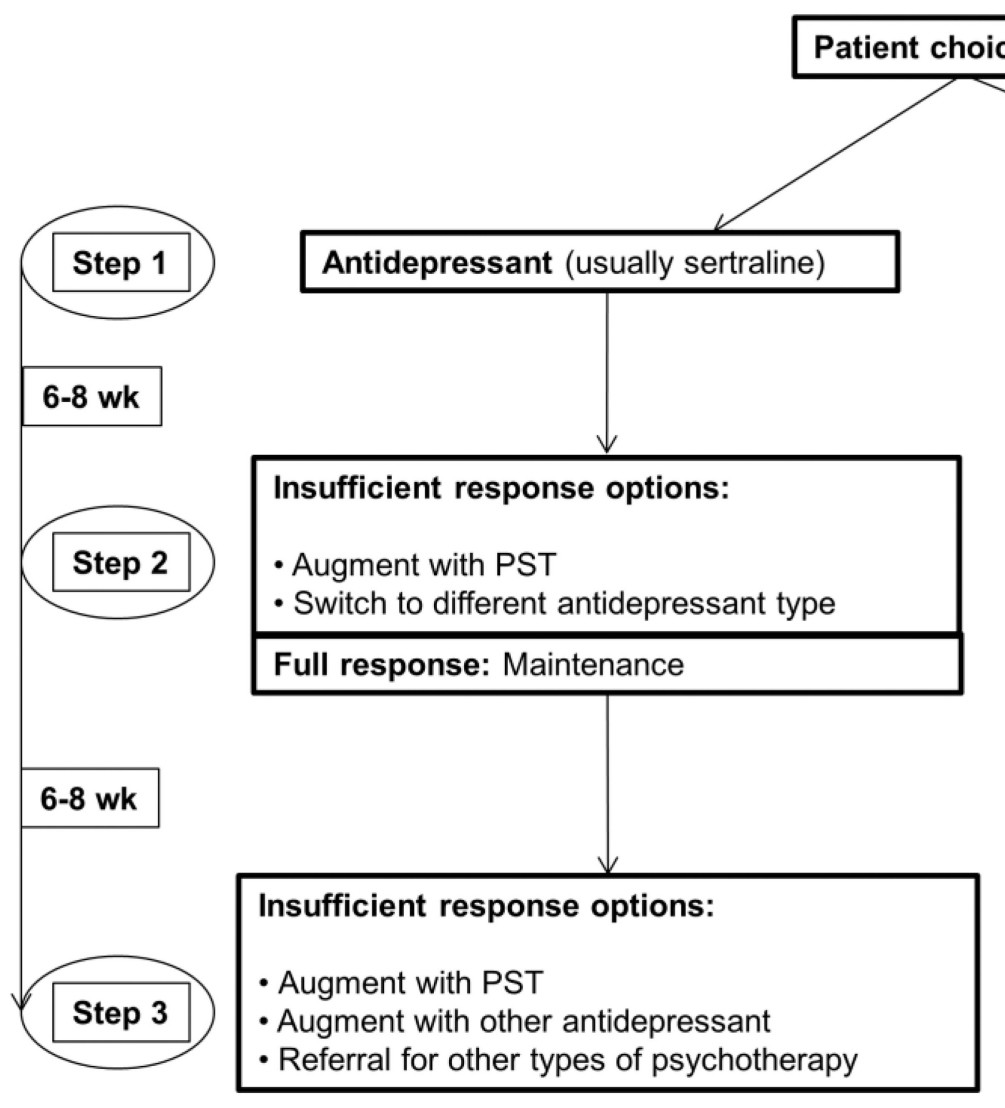

Full response: Maintenance

Figure 1.

Stepped Care Algorithm. 


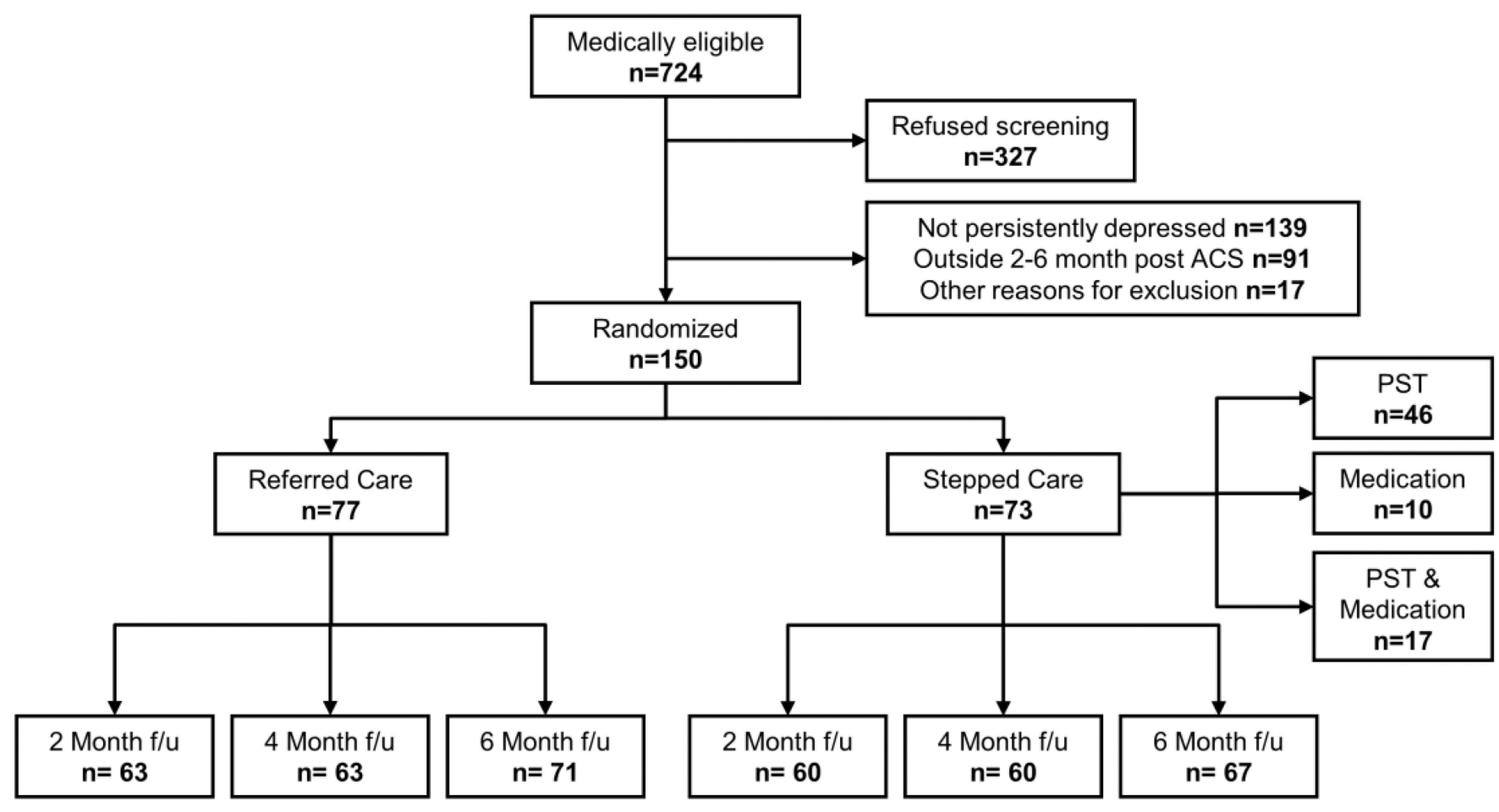

Figure 2.

Consort Diagram. Abbreviations: PST, problem solving therapy; ACS, acute coronary syndrome; f/u, follow-up. 
Table 1

Baseline demographic and clinical characteristics of 150 patients

\begin{tabular}{lc}
\hline Characteristic & No. $(\%)$ \\
\hline Average age, y & $59.6(10.4)^{a}$ \\
Women & $63(42.0)$ \\
Hispanic & $29(19.3)$ \\
Black & $50(33.3)$ \\
Education & \\
High school degree & $85(56.7)$ \\
College degree & $38(25.3)$ \\
Type of presenting ACS & \\
$\quad$ Unstable angina & $70(46.7)$ \\
Non-ST-segment MI & $37(24.7)$ \\
ST-segment MI & $40(26.7)$ \\
Bundle branch block or uncertain type of MI & $3(2.0)$ \\
Left ventricular ejection fraction $<0.45^{b}$ & $31(20.7)$ \\
BDI score $\geq 16$ at baseline & $102(68.0)$ \\
Ever prescribed antidepressant or antianxiety medication & \\
Use of antidepressant medication at time of enrollment ${ }^{c}$ & $85(56.7)$ \\
\hline
\end{tabular}

Abbreviations: ACS, acute coronary syndrome; BDI, Beck Depression Inventory; MI, myocardial infarction.

${ }^{a}$ Presented as average age (SD).

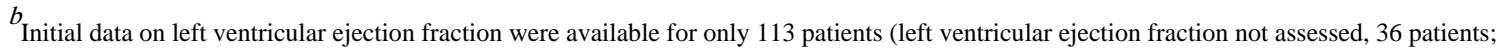
medical history forms not received, 1 patient).

$c_{\text {Based on self-report data }}$ 


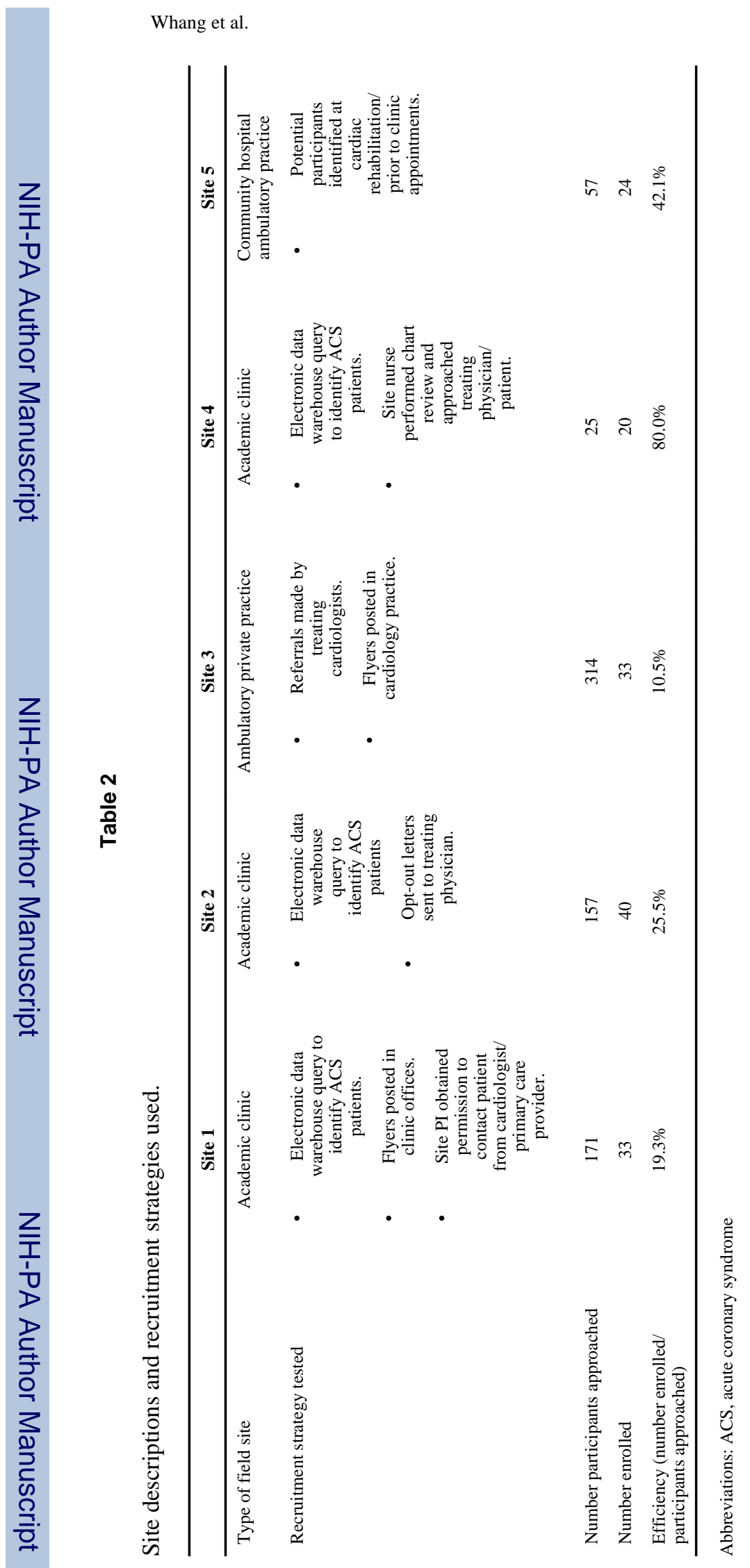

Contemp Clin Trials. Author manuscript; available in PMC 2013 September 01. 